\title{
Shedding light on photo-switchable analgesics for pain
}

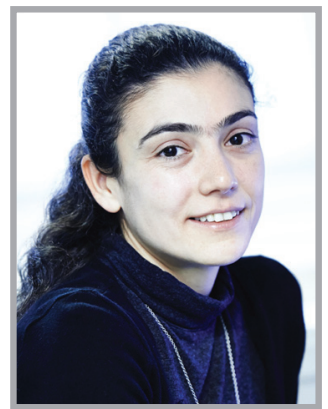

Parisa Gazerani*

First draft submitted: 27 August 2016; Accepted for publication: 7 October 2016; Published online: 25 October 2016
Photo-pharmacology [1] deals with photoresponsive agents that are compounds whose activity is controlled or regulated by light. Photo-responsiveness of these agents is achieved when a switch occurs between two (cis-trans) or more isoforms of photocompounds in response to light. Hence, the biological functions can be controlled by these compounds that act on native receptors or acceptors [1-3]. Two types of synthetic photo-switches exist, those that are covalently attached to their target (photoswitchable tethered ligands) and those lent interactions (photo-chromic ligands). Photo-switchable or photo-pharmaceutical terms are used to include both types. Targets for these compounds range from ion channels and G-protein-coupled receptors (GPCRs) to transporters, enzymes and lipids [2]. Photo-pharmacology principle holds an advantage of selectivity because light can be delivered with a high precision in terms of its intensity and wavelength for adjustable dosing [1-3]. The possibility to alter the activity of a drug by light offers several advantages, such as reducing that are tightly bound through noncova- off-target, systemic side effects or even drug resistance [3]. Light-controlled drugs have been further studied in the field of cancer, diabetes, microbial infections and neurology $[1-3]$. In the latter concept, only few photo-switchable regulators of nociception have been described so far, which will be presented here briefly to stimulate curiosity for further research.

\section{Photo-compounds for $\mu$-opioid receptor}

Photo-switchable opioids are compounds that can be reversibly switched on and off with light. Application of such compounds (potential photo-analgesics) can allow an optical control of $\mu$-opioid receptors. The first compound was an azobenzene derivative of fentanyl (photofentanyl-2 or PF2) [4]. Fentanyl is a potent synthetic $\mu$-opioid receptor agonist. The $\mu$-opioid receptor is a member of GPCRs (family A) that also include adrenergic receptors, muscarinic acetylcholine receptors, dopamine, histamine and serotonin receptors.

The GPCR's photo-switchable compound PF2 was produced and tested in human
*Department of Health Science \& Technology, Faculty of Medicine, Aalborg University, Frederik Bajers Vej 7A2-A2-208, 9220 Aalborg East, Denmark; Tel.: +45 9940 2412; gazerani@hst.aau.dk

\section{KEYWORDS}

- analgesics $\bullet$ anesthetics $\bullet$ pain

- photo-pharmaceutical

- photo-pharmacology

- photo-switchables 
"Photo-pharmacological control of nociception pain is a rapidly growing field." $\mu$-opioid receptor transfected into HEK293t cells with the G-protein-coupled inward rectifier channels (GIRK1 and GIRK2) [4]. The optimal photo-switching properties of $\mathrm{PF} 2$ were achieved at concentration of $25 \mathrm{~mm}$. Trans- $\mathrm{PF} 2$ is the isoform predominant in dark or in optical stimulation of 420-480 nm light. This isoform acted as an effective agonist for the $\mu$-opioid receptor. However, cis-PF2, the predominant isomer at $360 \mathrm{~nm}$ was almost inactive. Hence, switching from $360 \mathrm{~nm}$ to blue light initiated a quick potassium influx via GIRK channels that was terminated by $360 \mathrm{~nm}$ light, which could abolish the activation of $\mu$-receptors. Introduction of PF2 not only opened up a new road toward potential use of photo-analgesics in the future, but also to demonstrate that photo-switchable compounds can also be used to further investigate opioid receptors $[3,4]$.

\section{Photo-compounds for TRPV1 receptor}

Photo-switchable compounds acting on TRPV1 have also been developed [5]. TRPV1 is a nonselective cation channel with a well-established role in nociception. A group of researchers initiated a breakthrough investigation of optical control of TRPV1 and developed several photo-switchable compounds [5]. This group also presented the first example of fusion of photo-pharmacology and lipid signaling with potential application in controlling protein-lipid interactions [5] .

Capsaicin is a TRPV1 agonist. Azo derivatives of capsaicin were synthesized to investigate photoswitchable agonists of TRPV1. Isomeric (cis and trans) photo-switchable compounds were tested for their activity on TRPV1 channels. These compounds are inactive in dark or with blue light and active under UV-A light application. UV-switchable compounds have limited application for clinical use. Therefore, a lot of effort has been focused on synthesis of azo-benzenes that can be switched with visible light. One of the azologues of capsaicin (cis-AzCA4) was found to be the most effective in activating TRPV1. Photo-activation of TRPV1 with this compound was reversible and achieved in both HEK293T cells and C fibers. TRPV1-positive neurons of mice dorsal root ganglion selectively responded to cis-AzCA4. In vivo tests demonstrated a TRPV1-mediated hyperalgesia by this compound $[3,5]$.

\section{Photo-compounds for $\mathrm{GABA}_{A}$ receptors}

$\mathrm{GABA}_{\mathrm{A}}$ receptors are activated by GABA. GABA is an inhibitory neurotransmitter and following its interaction with $\mathrm{GABA}_{\mathrm{A}}$ receptors, chloride influx is promoted and the postsynaptic neuron is hyperpolarized. Hence, $\mathrm{GABA}_{\mathrm{A}}$ receptors have been targeted for producing anesthetic, hypnotic and anticonvulsant properties.

Propofol acts on $\mathrm{GABA}_{\mathrm{A}}$ and has long been used in systemic anesthesia. It is generally accepted that this lipophilic agent acts most likely through potentiation of GABA-induced currents and also through a direct activation of chloride channel when used at higher concentrations. $\mathrm{GABA}_{\mathrm{A}}$ receptors are not sensitive to light. A group of researchers [6] produced photoswitchable agents based on propofol molecular structure that could be switched between an active and an inactive form by the application of light. Azo-benzene derivatives of propofol were synthesized as azo-propofols 1-16 (AP1-16). Among these azo-propofols, AP2 presented favorable characteristics. Trans-AP2 was found capable of activating $\mathrm{GABA}_{\mathrm{A}}$ receptor subunit of $\alpha_{1} \beta_{2} \gamma_{2}$ in Xenopus oocytes. Cis isoform of this compound was found inactive. Whole-cell patch clamp method in HEK cells with trans-AP2 also showed that exposure to light leads to conversion of AP2 to its cis isoform and a decrease in current amplitude. Switching off the lights could reverse the diminished amplitude. An in vivo animal model in albino Xenopus laevis tadpoles was then applied to investigate AP2 anesthetic activity [6]. Inhibiting righting reflexes, a standard assay for studying anesthesia, was used and AP2 was shown to be capable of causing anesthesia. This study [6] demonstrated that azo-propofol AP2 potentiates GABA-induced chloride currents and can act as a light-dependent anesthetic agent, which is activated in dark and its activity is reversed by application of violet light [3,6]. It has been proposed that hyperpolarization-activated cation channels (HCN1) are also involved in propofol-induced anesthesia. AP2 might also be capable of modulating these targets, however, this is yet to be discovered [6].

\section{Photo-compounds for voltage-gated channels}

Quaternary ammonium-azobenzene-quaternary ammonium (QAQ) is a photo-switchable compound developed on the basis of lidocaine and its derivative QX-314, local anesthetics that block voltage-gated channels $[7,8]$. Trans-QAQ is capable of blocking ion channels, but the cis form is inactive. QAQ is selective for nociceptors and its inhibitory effect can be regulated by light. 
QAQ has been used together with capsaicin to block TRPV1-expressing nociceptors selectively. Under prolonged pain conditions, QAQ inhibitory effect does not seem to need a co-targeting of TRPV1, due to the fact that active nociceptive neurons would allow its internalization $[7,8]$. An in vivo test was also performed by topical application of QAQ to rats' cornea, where von Frey hair evoked responses demonstrated that transQAQ could block blink sensitivity following irradiation of $380 \mathrm{~nm}$ light [8].

Fotocaine is another compound based on azologization of fomocaine, which is a local anesthetic. A group of researchers [9] have demonstrated that fotocaine is taken up by neurons in brain slices where neuronal action potential can be regulated by application of light. Neurophysiological application of this photoswitchable compound has opened up a possibility to test its application as a potential analgesic [9].

\section{Perspectives}

Photo-pharmacology deals with a delicate process of design, synthesis and application of drugs that can be modulated with light. This approach offers a possibility to solve off-target side effects. Photo-switchable compounds are bioactive molecules with moieties that change their structure by light and these changes regulate their therapeutic action. Photo-pharmacological control of nociception pain is a rapidly growing field. More photo-switchable compounds are in pipeline, for example, photo-switchable compounds for ionotropic glutamate receptors, kainate receptors, AMPA receptors, metabotropic glutamate receptors, adrenergic receptors, muscarinic acetylcholine receptors, dopamine, histamine, serotonin receptors, calcium and potassium channels and number of transporters and pumps [2,3]. Innovative photo-pharmaceutical candidates would not be achieved without combination of diverse expertise such as chemistry, biology, physiology, pharmacology and medicine [2] Suitable photo-switchable compounds and targets need to be chosen. Under ideal conditions, a photo-switchable compound is active as an agonist or antagonist in one molecular structure and fully inactive in other. A systematic classification of body organs has been proposed [3] to make it easier to understand how light can be delivered to them and this needs to be implemented for the compounds under developments. This classification has identified class 1 for easily accessible organs such as skin and eye. Class 2 compounds are those that can be accessed by endoscopy, for example, GI tract, intraoral cavity, sinuses, respiratory system, cervix and bladder. Class 3 includes those parts that are accessible through skin but without any incision, for example, thyroid, testicles, lymph nodes, muscles and tissues lying just under the skin. Class 4 would accommodate compounds that need a minor incision for accessibility, for example, pancreas, liver, ovaries, stomach, intestines, kidneys, spleen, prostate, some blood vessels, glands, lymph nodes, muscles and bones. Major incision or operative procedure would be needed for class 5 , for example, to reach brain and bone marrow. Besides delivery, testing and ensuring efficacy and safety of developed compounds, economic aspects of development must also be taken into account for further development of realistic choices toward their clinical use [1-3].

\section{Financial \& competing interests disclosure}

The author has no relevant affiliations or financial involvement with any organization or entity with a financial interest in or financial conflict with the subject matter or materials discussed in the manuscript. This includes employment, consultancies, honoraria, stock ownership or options, expert testimony, grants or patents received or pending, or royalties.

No writing assistance was utilized in the production of this manuscript

\section{References}

Papers of special note have been highlighted as: •• of considerable interest

1 Velema WA, Szymanski W, Feringa BL. Photopharmacology: beyond proof of principle. J. Am. Chem. Soc. 136(6), 2178-2191 (2014).

2 Broichhagen J, Frank JA, Trauner D. A roadmap to success in photopharmacology. Acc. Chem. Res. 48(7), 1947-1960 (2015).
- An excellent paper giving directions for future investigation.

3 Lerch MM, Hansen MJ, van Dam GM, Szymanski W, Feringa BL. Emerging targets in photopharmacology. Angew. Chem. Int. Ed. Engl. 55(37), 10978-10999 (2016).

-• An excellent recent review on targets in photo-pharmacology.

4 Schönberger M, Trauner D. A photochromic agonist for $\mu$-opioid receptors. Angew.
Chem. Int. Ed. Engl. 53(12), 3264-3267 (2014).

5 Frank JA, Moroni M, Moshourab R, Sumser M, Lewin GR, Trauner D. Photoswitchable fatty acids enable optical control of TRPV1. Nat. Commun. 6, 7118 (2015).

6 Stein M, Middendorp SJ, Carta V et al. Azo-propofols: photochromic potentiators of GABA(A) receptors. Angew. Chem. Int. Ed. Engl. 51(42), 10500-10504 (2012). 


\section{EDITORIAL Gazerani}

7 Mourot A, Tochitsky I, Kramer RH. Light at the end of the channel: optical manipulation of intrinsic neuronal excitability with chemical photoswitches. Front. Mol. Neurosci. 6, 5 (2013). rot A, Fehrentz I, Le Feuvre Y et al. Rapid optical control of nociception with an ion-channel photoswitch. Nat. Methods 9(4), 396-402 (2012).
9 Schoenberger M, Damijonaitis A, Zhang Z, Nagel D, Trauner D. Development of a new photochromic ion channel blocker via azologization of fomocaine. ACS Chem. Neurosci. 5(7), 514-518 (2014). 